\title{
Corona Immunitas: study protocol of a nationwide program of SARS- CoV-2 seroprevalence and seroepidemiologic studies in Switzerland
}

\author{
Erin A. West ${ }^{1} \cdot$ Daniela Anker $^{2} \cdot$ Rebecca Amati $^{3} \cdot$ Aude Richard $^{4,5} \cdot$ Ania Wisniak $^{4,5} \cdot$ Audrey Butty $^{6}$. \\ Emiliano Albanese $^{3} \cdot$ Murielle Bochud $^{6}$ - Arnaud Chiolero ${ }^{2,7,8} \cdot$ Luca Crivelli $^{3,9}$ - Stéphane Cullati ${ }^{2,10}$. \\ Valérie d'Acremont ${ }^{6,11,12}$. Adina Mihaela Epure ${ }^{2,6} \cdot$ Jan Fehr $^{1}$ - Antoine Flahault ${ }^{5}$. Luc Fornerod ${ }^{13}$. \\ Irène Frank ${ }^{14}$ - Anja Frei ${ }^{1}$ - Gisela Michel ${ }^{15}$. Semira Gonseth ${ }^{6}$. Idris Guessous ${ }^{4}$ - Medea Imboden ${ }^{11,12}$. \\ Christian R. Kahlert ${ }^{16,17}$ - Laurent Kaufmann ${ }^{18}$. Philipp Kohler ${ }^{16}$. Nicolai Mösli ${ }^{11,12}$ - Daniel Paris ${ }^{11,12}$. \\ Nicole Probst-Hensch ${ }^{11,12}$ - Nicolas Rodondi ${ }^{7,19}$ - Silvia Stringhini ${ }^{4,6} \cdot$ Thomas Vermes $^{11,12}$. \\ Fabian Vollrath ${ }^{20}$. Milo A. Puhan ${ }^{1}$ on behalf of the Corona Immunitas Research Group
}

Received: 23 August 2020/Revised: 16 September 2020/ Accepted: 16 September 2020 / Published online: 24 October 2020

(C) The Author(s) 2020

\begin{abstract}
Objectives Seroprevalence studies to assess the spread of SARS-CoV-2 infection in the general population and subgroups are key for evaluating mitigation and vaccination policies and for understanding the spread of the disease both on the national level and for comparison with the international community.

Methods Corona Immunitas is a research program of coordinated, population-based, seroprevalence studies implemented by Swiss School of Public Health (SSPH+). Over 28,340 participants, randomly selected and age-stratified, with some regional specificities will be included. Additional studies in vulnerable and highly exposed subpopulations are also planned. The studies will assess population immunological status during the pandemic.

Results Phase one (first wave of pandemic) estimates from Geneva showed a steady increase in seroprevalence up to $10.8 \%$ (95\% CI 8.2-13.9, $n=775$ ) by May 9, 2020. Since June, Zurich, Lausanne, Basel City/Land, Ticino, and Fribourg recruited a total of 5973 participants for phase two thus far.

Conclusions Corona Immunitas will generate reliable, comparable, and high-quality serological and epidemiological data with extensive coverage of Switzerland and of several subpopulations, informing health policies and decision making in both economic and societal sectors.
\end{abstract}

ISRCTN Registry: https://www.isrctn.com/ISRCTN18181860.

Keywords Prevalence $\cdot$ Serosurvey $\cdot$ Longitudinal $\cdot$ SARS-CoV-2 Socioeconomic differences $\cdot$ Hygiene practices

Erin A. West and Daniela Anker have contributed equally to this work.

This cohort study has been deemed a "Category A" research project according to HRO Art. 7, as the planned data collection entails only minimal risks and burden to patients. Other seroprevalence studies of SARS-COV-2-antibodies under the umbrella of Corona Immunitas by SSPH+, following mainly the same protocol, have already been approved by the local ethics commissions and already started in the Canons of Geneva (SEROCoV-POP study; Project Leader Dr. Silvia Stringhini, BASEC No 2020-00881), Ticino (Corona Immunitas Ticino; Project Leader Prof. Dr. Emiliano Albanese, BASEC No 2020-01514) and Vaud (UnderCOVer study; Project Leader Prof. Valérie
D'Acremont, BASEC No 2020-00887). In the Cantons Basel Landschaft and Basel Stadt, the project has been submitted to the local ethics commission (CoV-Co-Basel, Project Leader Prof. Dr. Nicole Probst-Hensch, BASEC No 2020-00927).

Electronic supplementary material The online version of this article (https://doi.org/10.1007/s00038-020-01494-0) contains supplementary material, which is available to authorized users.

Milo A. Puhan

miloalan.puhan@uzh.ch

Extended author information available on the last page of the article 


\section{Introduction}

By August 21, 2020, over 22,000,000 persons were diagnosed with a severe acute respiratory syndrome coronavirus-2 (SARS-CoV-2) (Gorbalenya et al. 2020) infection globally and the number of deaths exceeded 790,000 (Worldometer 2020). Switzerland alone had over 39,000 confirmed cases with over 1700 deaths (Federal Office of Public Health (FOPH) 2020a). The 2019 novel coronavirus (COVID-19) pandemic has caused worldwide lockdowns and restrictions on individuals' freedom of movement to limit the spread of this virus. The measures taken by the Swiss government aimed to balance the protection of the population against SARS$\mathrm{CoV}-2$ and the functioning of the economy and society at large. As the number of cases rose in March 2020, a containment strategy of contact tracing and isolation was not enough (Hellewell et al. 2020) and the country was quickly put into a semi-lockdown. Non-essential businesses were closed, citizens were asked to stay at home, and distance learning for all schools was implemented (FOPH 2020b). Later, with the flattening of the pandemic curve and increasing political pressure, the semi-lockdown was progressively lifted. These decisions had, and still have, to be taken, while many epidemiologic features of the SARS-CoV-2 pandemic are unknown, such as transmission characteristics of the virus, prevalence of infection, extent of immunity after infection and its relationship to disease symptoms and severity, and how different groups of the population are affected. To overcome this, it is crucial to monitor the pandemic and increase our knowledge about the virus with solid data to inform future policy decisions and help prepare potential future outbreak responses in a balanced manner (Arora et al. 2020).

Seroprevalence surveys are designed to assess the proportion of populations infected by the virus, where diagnostic testing fails to ascertain the full scale of the spread due to unreported and asymptomatic cases. However, weaknesses and between-study differences in methodology limit their reliability and comparability. A rapid, systematic review of early seroprevalence surveys by Bobrovitz et al. (preprint) on SARS-CoV-2 came out identifying 73 completed and ongoing seroprevalence studies across the world. These studies reported seroprevalence estimates ranging between 0.4 and 59.3\%. The review found that, of the 23 studies reporting prevalence estimates, many had a moderate or high risk of bias (Bobrovitz et al. 2020; Joanna Briggs Institute 2016). Reportedly, the largest issues with these early studies included inadequate sampling methods and antibody test performance, lack of or inconsistent use of questionnaire assessments, and varying designs and analyses, hindering comparison of results.

To overcome these limitations and generate information about the SARS-CoV-2 pandemic that is accurate and generalizable to the whole population, population-based seroprevalence studies with reliable methodologies are needed (Pollán et al. 2020). It is essential to include strong coordination across study sites and to ensure cross-regional comparability. Standardized methodologies, including the same antibody tests, questionnaires, and study procedures, are vital to obtain reliable estimates within a country (World Health Organization (WHO) 2020). Further, an additional understanding of the duration of immunity, demographic differences, and the economic and psychological impact of the pandemic and subsequent control measures adds more depth and understanding of the overall situation. This further helps inform policy-making and provides better preparedness, both logistically and with respect to the economic consequences, for any future outbreaks.

We describe here the protocol of Corona Immunitas, a centrally coordinated research program consisting of repeated cross-sectional and longitudinal seroprevalence and seroepidemiological studies conducted across several regions and populations in Switzerland, whose aim is to generate reliable data to inform policy-making.

\section{Study objectives}

The main goal of the Corona Immunitas research program is to determine the extent and nature of infection with SARS-CoV-2 in Switzerland in a highly consistent and comprehensive way, first, in the general population and, second, in vulnerable and highly exposed subpopulations. Specific aims are to: (1) estimate the number of individuals infected with SARS-CoV-2 in the population with or without symptoms at several points in time; (2) compare the seroprevalence between the general population and specific subpopulations; (3) investigate the characteristics, duration, and extent of immunity after infection; (4) assess the association between participant characteristics and behaviors with their risk of infection; and (5) quantify the association between the pandemic and participants' mental and physical health. Most importantly, this evidence-based program aims to provide policy-makers and other decision makers with important evidence for deciding which public health and setting-specific measures to implement or lift in the general population and specific subpopulations at different points in time.

\section{Methods}

The protocol has been developed according to the Consortium for the Standardization of Influenza Seroepidemiology (CONSISE) Statement on the Reporting of 


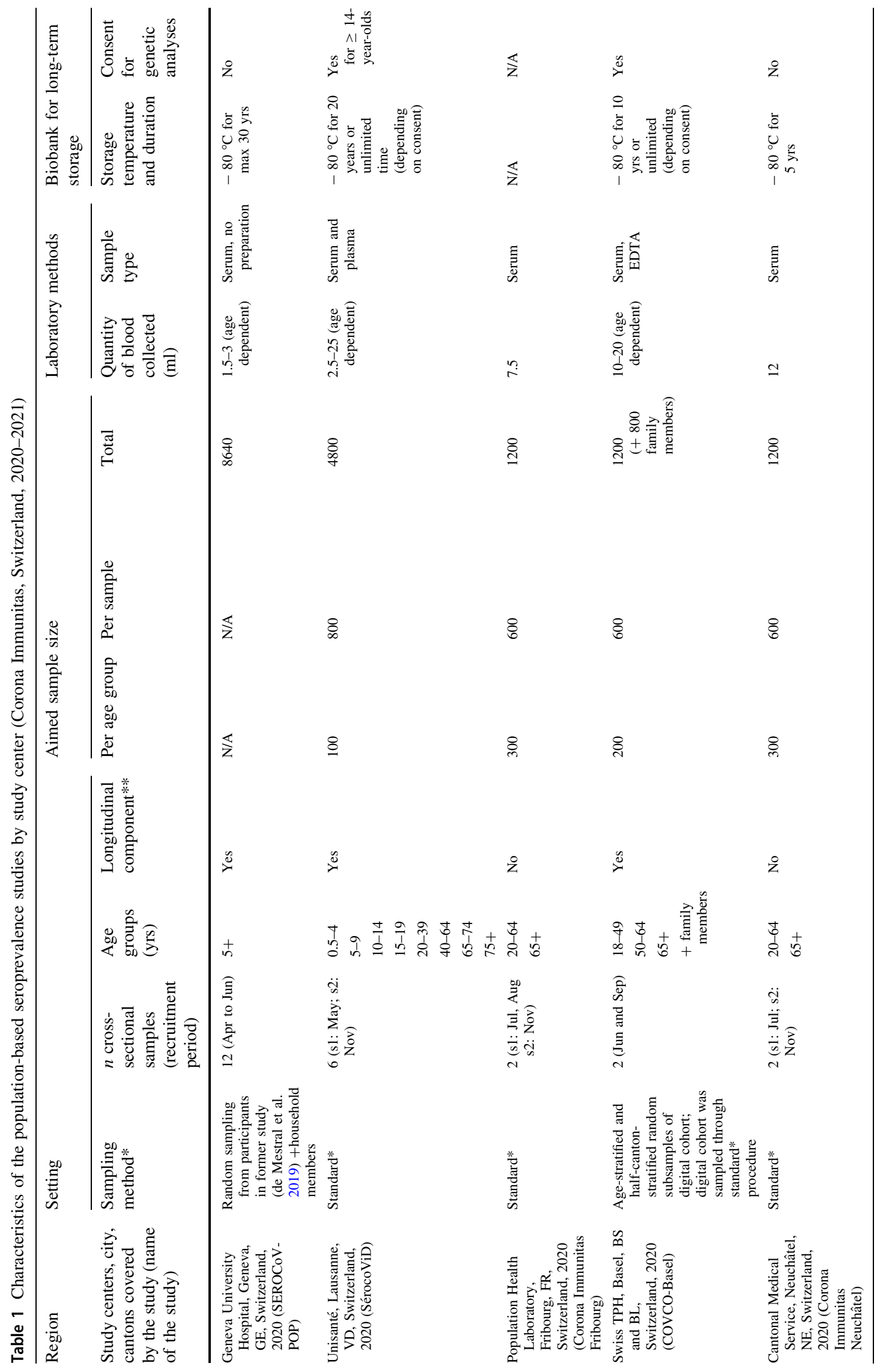




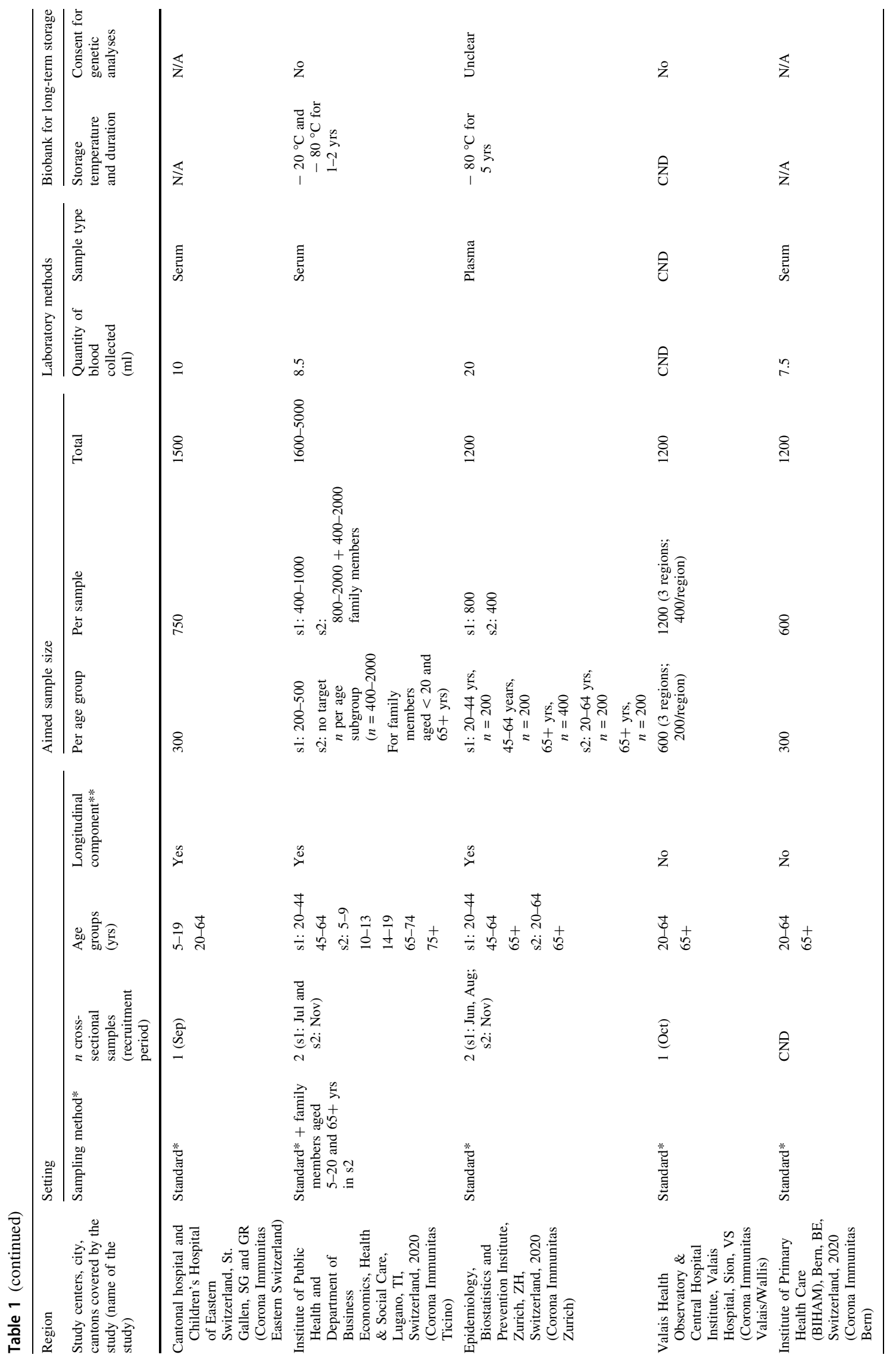




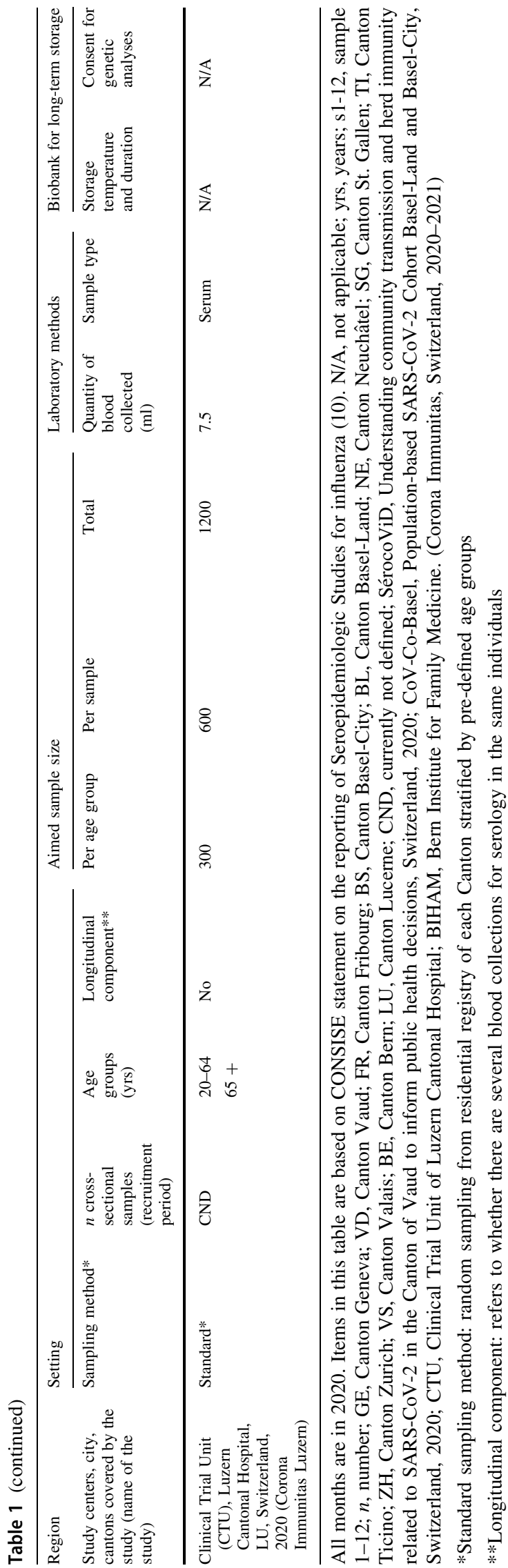

Seroepidemiologic Studies for Influenza (ROSES-I) (Joanna Briggs Institute 2016).

\section{Study design and phases of the Corona Immunitas program}

The Corona Immunitas program includes more than 20 cross-sectional and longitudinal studies in the general population and in specific subpopulations (Tables 1 and 2) with serological testing at baseline and a digital only or combined digital and serological follow-up. The questionnaires and the antibody test are standardized across the sites to guarantee comparability across the country. The Corona Immunitas research group shares protocols for all the studies in an open science way in order to exchange knowledge and expertise, to create synergisms but also to reduce redundancies across cantons and regions. We define our general, population-based studies as seroprevalence studies and our subpopulation studies as seroepidemiological studies per the lexicon defined by Horby et al. (2017).

The studies are conducted in a series of repeated phases, the timing of which is subject to change according to the highly dynamic pandemic and additional needs that may emerge (Fig. 1). The first phase began in April 2020 and included early estimates of seroprevalence during the first wave from Geneva (Stringhini et al. 2020). The second phase includes estimates of seroprevalence across Switzerland during summer, after the first peak of the pandemic. This phase, conducted as recommended by the WHO protocol (WHO 2013) after the (first) pandemic wave, includes highly (Cantons of Geneva, Vaud and Ticino), moderately (Cantons of Fribourg, Neuchatel and Basel City/Land) and little affected regions of Switzerland (Cantons of Zurich and St. Gallen) (FOPH 2020a). The third phase will be in the fall and includes estimates of seroprevalence across Switzerland about 4-5 months after the lifting of lockdown measures. This phase will help evaluate the quality of the monitoring systems in place and determine the needs for a vaccination program. A fourth phase will cover March 2021 and will evaluate the measures after the winter, as well as the completeness and duration of immunity.

\section{General population seroprevalence studies}

Geneva was the first canton to enroll randomly selected participants of a population-based study (Bus Santé) ongoing since 1993 (Stringhini 2020). For the other Cantons, the Federal Statistical Office (FSO) randomly selected, per region, potential participants from the residential registry. The number of randomly chosen participants takes into consideration the net sample size (i.e., target sample 


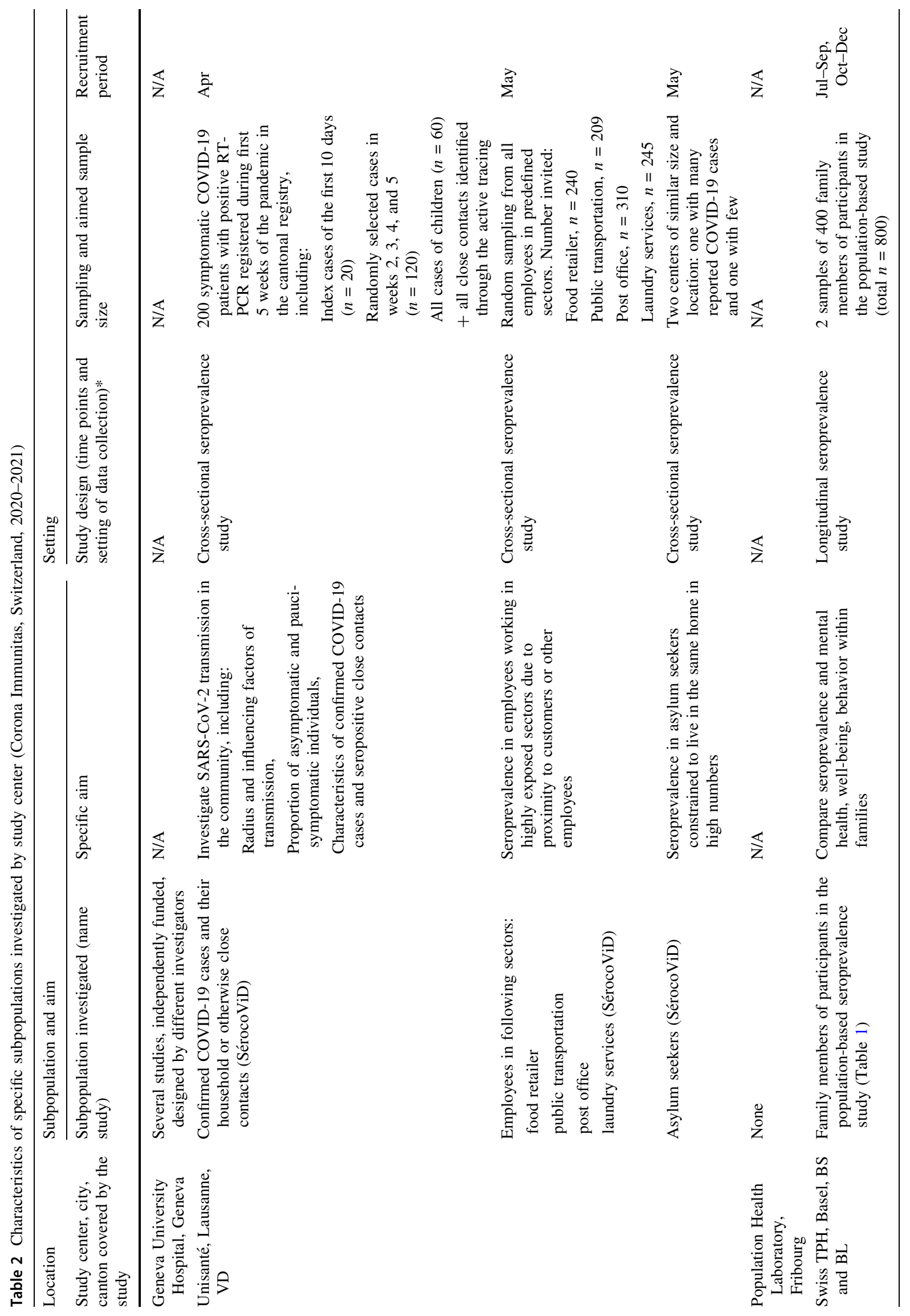




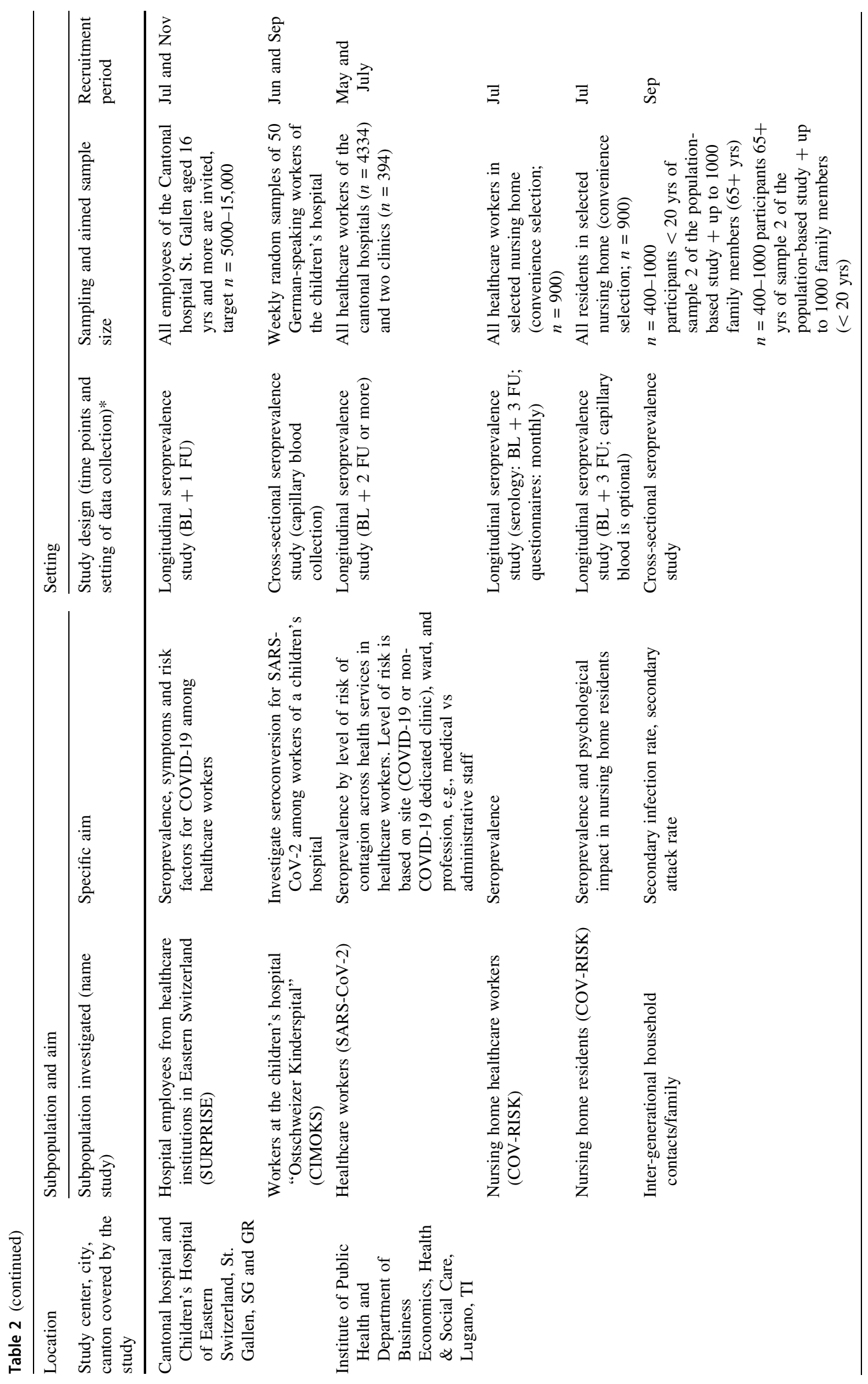




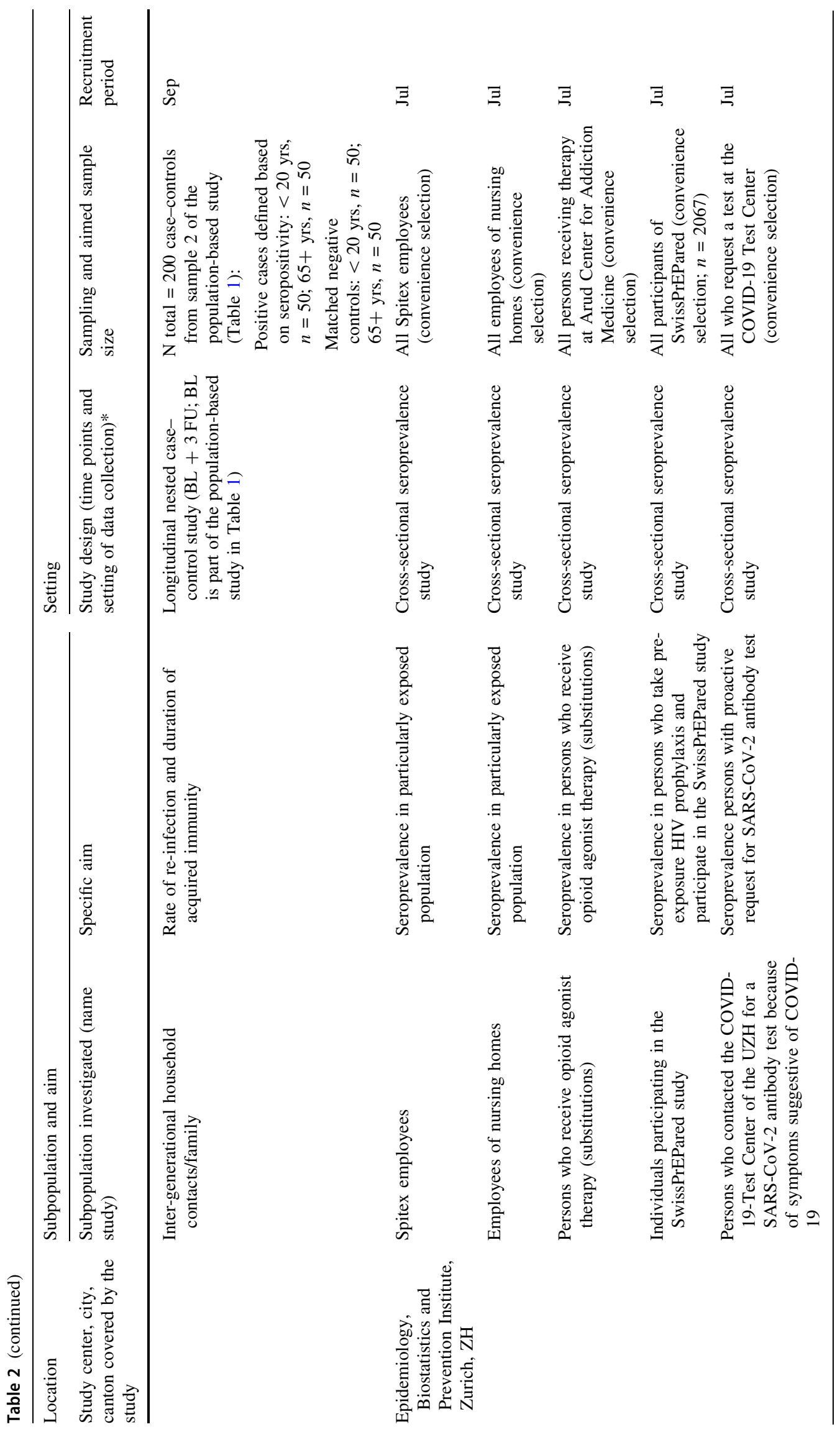




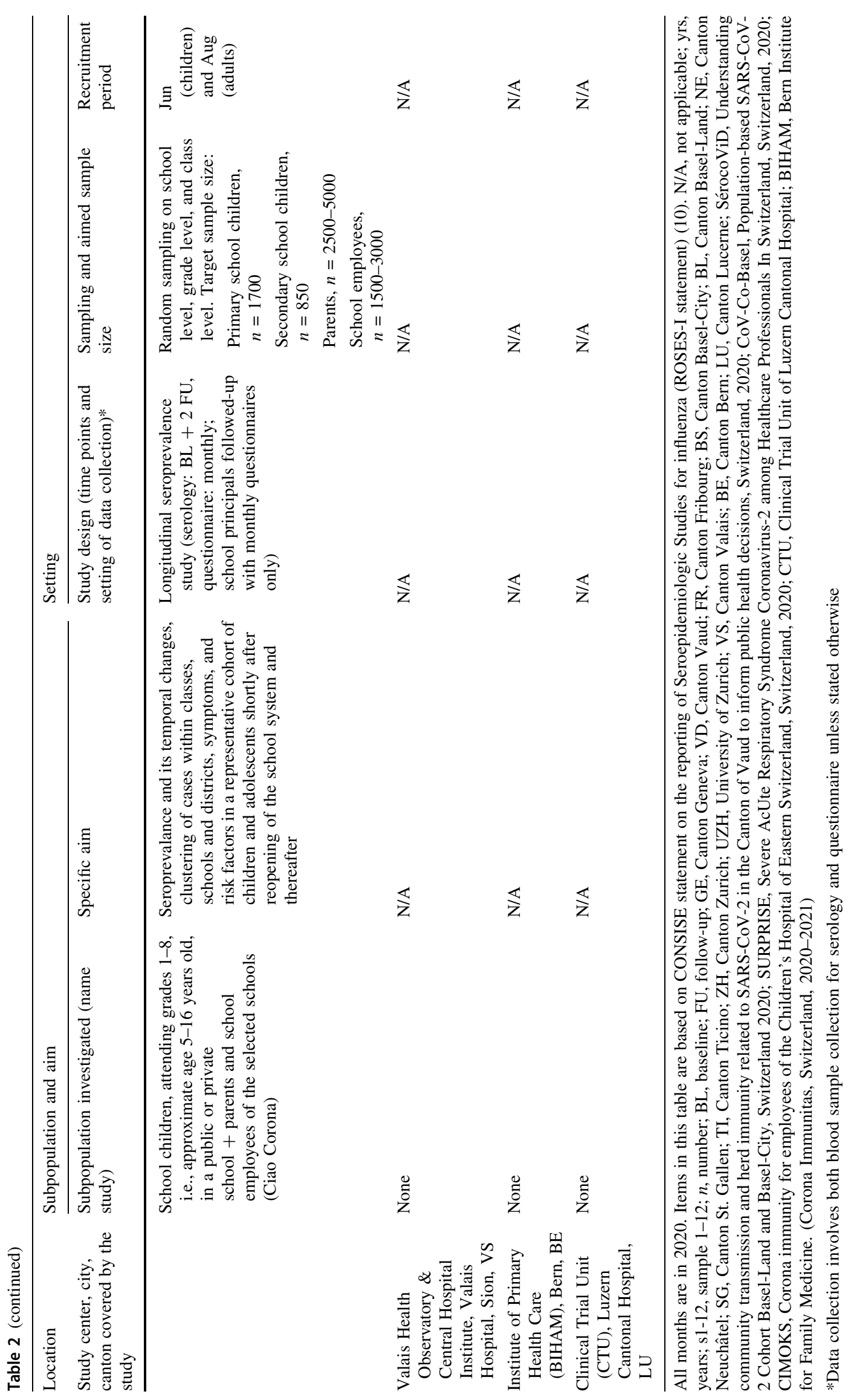




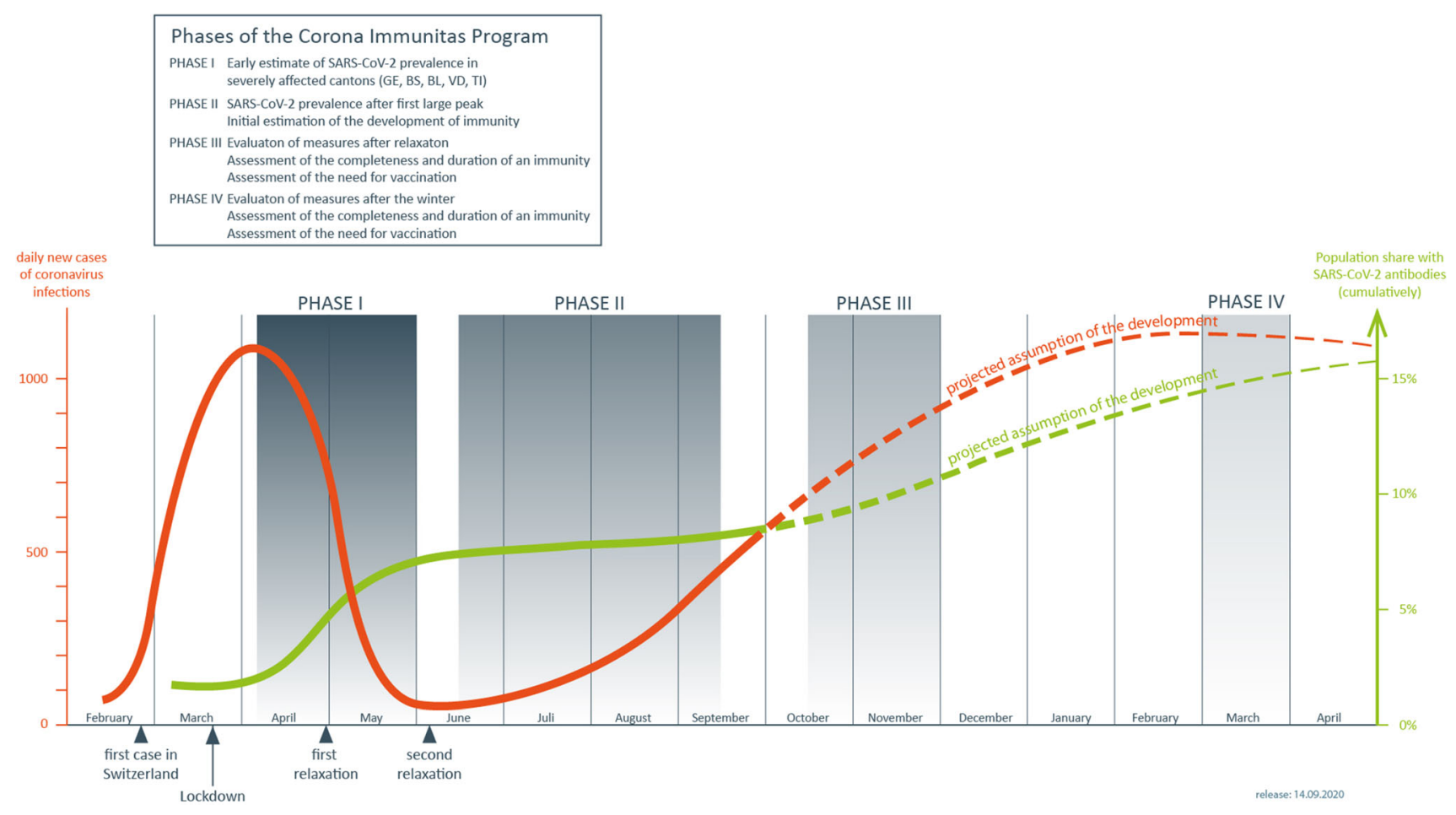

Fig. 1 Phases of the pandemic as defined in the Corona Immunitas program (Switzerland 2020-2021)

size) as well as the expected participation rate and reserve samples (residents selected in the case that more participants are needed to fulfill the sample size requirements). The FSO provides all participating Cantons with a list of participants, including their full name, postal address and preferred language. The sampling is age-stratified into three age groups: 5-19 years, 20-64 years, and 65 years and older, though some Cantons have also chosen to recruit younger participants. Cantons can choose slightly modified sampling procedures, such as recruiting participants' family or household members (Table 1).

The inclusion criteria for the random sampling are residency in one of the participating Cantons. "Vulnerable to COVID-19 persons" (detailed below) are included. For inclusion in the data collection, individuals with a suspected or confirmed acute SARS-CoV-2 infection are postponed for a visit to $48 \mathrm{~h}$ after disappearance of symptoms or 21 days after a positive SARS-CoV-2 reverse transcription polymerase chain reaction test (RT-PCR) result. Excluded from the residential registry of the FSO are diplomats, persons with a foreign address in the registry, persons in asylum procedure, persons with a shortterm residence permit, and elderly people in nursing homes.

If selected, "vulnerable to COVID-19 persons," i.e., persons at risk of a severe course of SARS-CoV-2 infection, can participate. They are defined in accordance with the recommendations by the Swiss Federal Office of Public Health (FOPH) (FOPH 2020c). The handling of those participants vulnerable to COVID-19 varies depending on the site. Persons are considered at risk of a severe course of SARS-CoV-2 infection if they are 65 years of age or older, pregnant or if they have the following conditions: diabetes; cardiovascular disease; chronic diseases of the respiratory tract; immune weaknesses due to disease or therapy; cancer(s); and obesity defined by a body mass index (BMI) $>30 \mathrm{~kg} / \mathrm{m}^{2}$ for adults and above the 97 th percentile of Swiss BMI growth curves for children and adolescents (https://www.paediatrieschweiz.ch). While the FOPH considers persons with high blood pressure to be at risk, the Swiss Hypertension Society recommends applying the same precautionary measures for hypertensive patients as for the rest of the population (Swiss Society of Hypertension 2020).

\section{Subpopulation seroepidemiological studies}

Corona Immunitas also includes specific subpopulations deemed especially exposed or vulnerable and about which more knowledge is necessary to inform policy- and decision making. Each center decided which subpopulation should be targeted based on the needs of stakeholders (e.g., Cantonal health authorities) or scientific interest. These studies follow the same study protocol as the general 


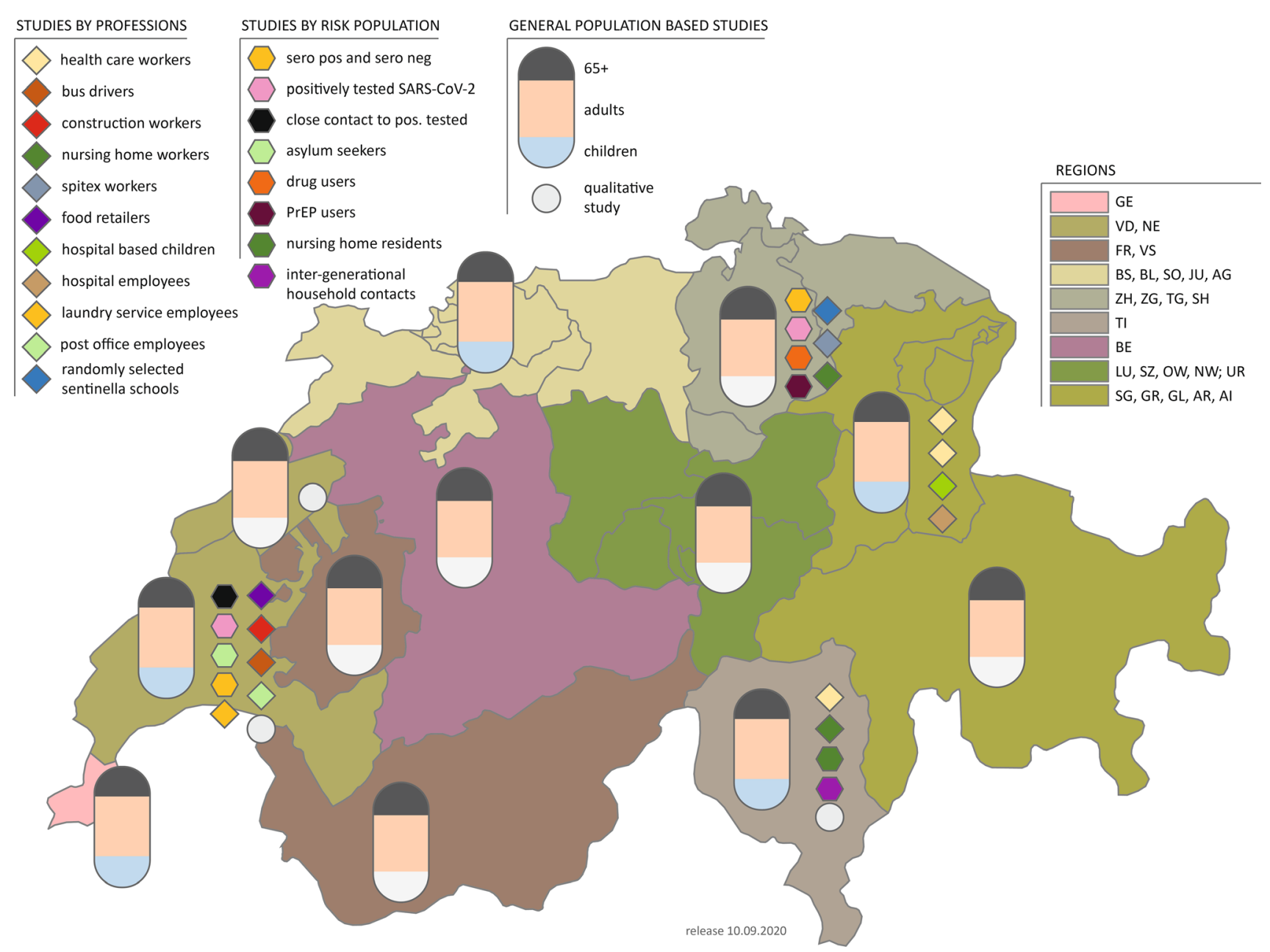

Fig. 2 Overview of seroprevalence studies in Switzerland (Corona Immunitas 2020-2021). ZH Zurich, BE Bern, $L U$ Luzern, $U R$ Uri, SZ Schwytz, $O W$ Obwald, $N W$ Nidwald, GL Glarus, ZG Zug, FR Fribourg, SO Solothurn, BS Basel-City, $B L$ Basel-Land, $S H$ Schaffhausen, $A R$ Appenzell Ausserrhoden, $A I$ Appenzell Innerrhoden, $S G$ St. Gallen, GR Graubünden, $A G$ Aargau, $T G$ Thurgau, TI
Ticino, $V D$ Vaud, VS Valais, $N E$ Neuchâtel, $G E$ Geneva, $J U$ Jura, $\operatorname{PrEP}$ Pre-exposure prophylaxis for HIV prevention. See Tables 1 and 2 for a detailed description of the studies. (Corona Immunitas, Switzerland, 2020-2021). *Children study with white bottoms instead of blue bottoms means there is no population-based children's study

online. Feedback of the serology test to participants is handled individually by the sites and subject to variations.

\section{Baseline assessment}

An example of the full study flow is outlined in Fig. 3 . Informed written or electronic consent is obtained before any procedure of the study visit. Participants can fill the baseline questionnaire online or use a paper form. The questionnaire takes approximately $20 \mathrm{~min}$ to complete and includes demographic questions, symptoms, other tests taken for SARS-CoV-2, preventative measure behaviors, and quality of life measures. Details of the questions asked of the participants are given in Table 3. The full questionnaires used nationwide are published in the online supplementary material. 


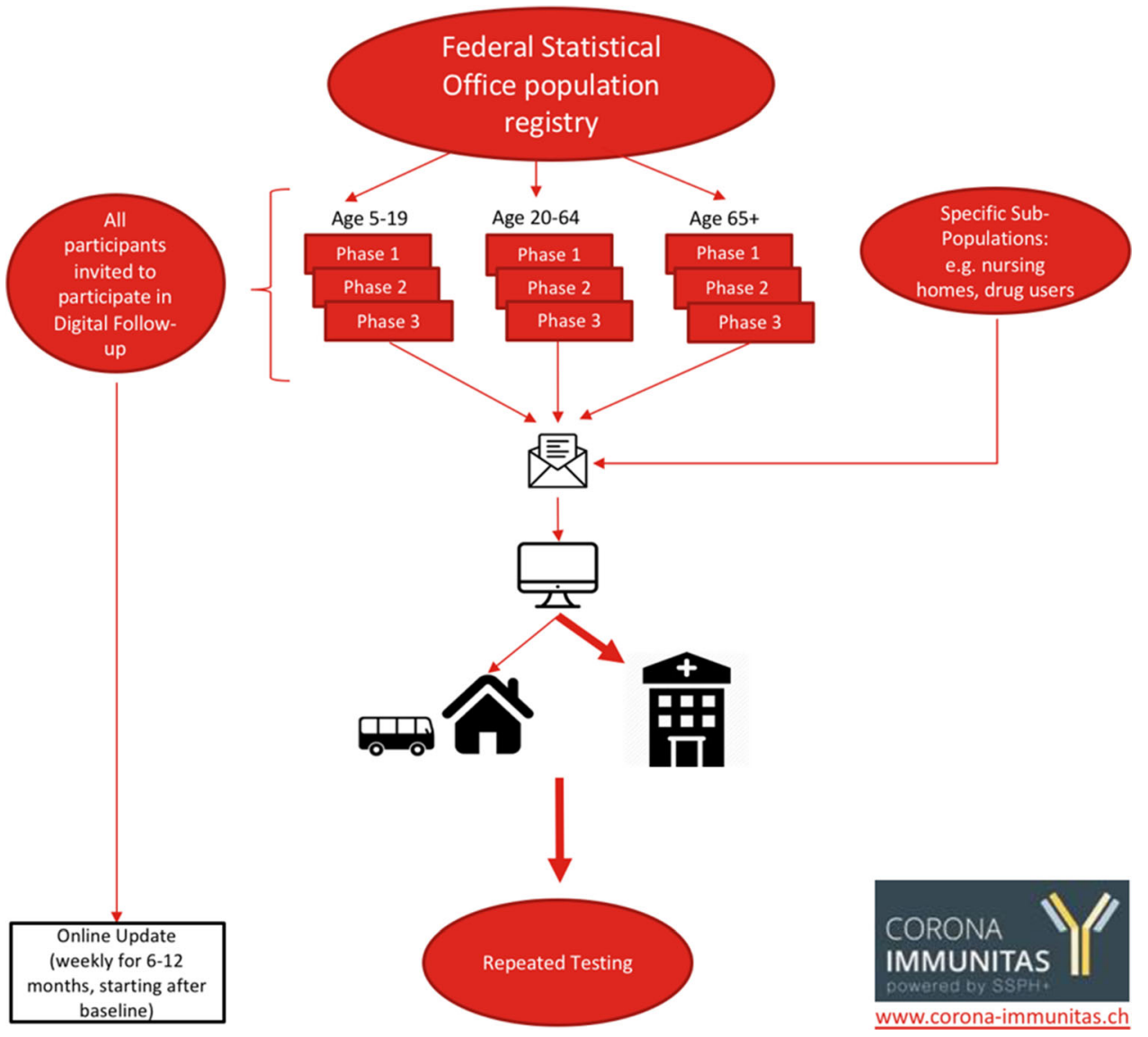

Fig. 3 Example study flow of seroprevalence studies (Corona Immunitas, Switzerland, 2020-2021)

Venous blood sampling is performed at a blood collection site or at home. All staff have access to the necessary infrastructure for blood withdrawal and safe storage of biological samples. The entire process follows Standardized Operating Procedures (SOPs). Blood drawing is performed by trained healthcare staff (i.e., nurse, assistant nurse, or junior doctor). The quantity of blood varies according to the study site, and depending on whether additional analyses are performed. Standard hygiene rules are followed such as usual handwashing, disinfection procedures, and wearing of masks and gloves. All participants are required to wear a mask, provided by the study team, during all interactions.

Samples are transported to a local laboratory or the Vaud Central University Hospital (CHUV) directly or centrifuged before transport to the laboratory if possible, aliquoted and stored in a biobank on each site at $-20{ }^{\circ} \mathrm{C}$ or $-80{ }^{\circ} \mathrm{C}$ before the serological test. Samples are delivered within $16 \mathrm{~h}$ of being taken. Team members are trained in safe management practices and procedures for contamination accidents. Serum is prepared with serum gel and plasma with ethylenediaminetetraacetic acid (EDTA) before the serology test. Depending on the site, serum or plasma from the drawn venous blood is analyzed for the presence of SARS-CoV-2 IgG and IgA antibodies. Some study sites will store additional serum, saliva or plasma samples in a biobank for further use in this or in other studies; genetic and epigenetic analyses are planned in several centers. Participants are informed about the planned analyses and provide broad consent for future research use of biospecimens.

The study data are collected and managed using REDCap electronic data capturing tools (Harris et al. 2019) hosted at the responsible universities. Websites of study centers are fully detailed in the supplementary material Table S1.

\section{Selection of antibody tests}

The selection of a common test followed a stepwise procedure. We developed a set of criteria (see supplementary material Table S2) that refer to the nature of the test, results from validation studies and the logistics and cost of the test. Members of the Corona Immunitas consortium 
Table 3 Example of schedule of assessments (Corona Immunitas, Switzerland, 2020-2021)

\begin{tabular}{|c|c|c|c|}
\hline Contacts & $\begin{array}{l}\text { Invitation/ } \\
\text { recruitment }\end{array}$ & $\begin{array}{l}\text { Study visit } \\
\text { (BL) }\end{array}$ & Digital follow-up \\
\hline Timing & Prior to Day 0 & Day 0 & Week 1 to week 52 (weekly) \\
\hline Study invitation & $\mathrm{X}$ & & \\
\hline Invitation for Baseline data collection (and visit—depending on center) & $\mathrm{X}$ & & \\
\hline Confirmation letter or email, written information & $\mathrm{X}$ & & \\
\hline Baseline questionnaire (online): & $\mathrm{X}$ & $X$ & \\
\hline Personal demographics \& health data & & & \\
\hline $\begin{array}{l}\text { COVID-19 specific data; including symptoms, hospitalization, relevant } \\
\text { medications, other SARS-CoV-2 tests }\end{array}$ & & & \\
\hline Socio-demographic data & & & \\
\hline Economic impact due to lockdown measures & & & \\
\hline Persons in immediate vicinity and their relevant symptoms & & & \\
\hline Preventive measures, exposure and level of concern over pandemic & & & \\
\hline Oral and/or written information & & $\mathrm{X}$ & \\
\hline Check inclusion-/ exclusion criteria & & $\mathrm{X}$ & \\
\hline Written consent & & $\mathrm{X}$ & \\
\hline Blood sampling & & $X$ & \\
\hline Information on test interpretation & & $\mathrm{X}$ & \\
\hline Information on digital follow-up & & $\mathrm{X}$ & \\
\hline Information on further procedure according to subpopulation & & $X$ & \\
\hline Weekly digital follow-up questionnaire (online): & & & $\mathrm{X}$ \\
\hline $\begin{array}{l}\text { Symptoms, healthcare professional contacts, hospitalizations, test results, } \\
\text { preventive measures }\end{array}$ & & & \\
\hline Monthly digital follow-up questionnaire (online): & & & $\mathrm{X}$ \\
\hline $\begin{array}{l}\text { Access to health care, behavior, daily activities, mental health, well-being, } \\
\text { usage of SwissCovid App (FOPH 2020d), motivation to participate in study }\end{array}$ & & & \\
\hline
\end{tabular}

$B L$ baseline

independently rated the tests that were submitted for use in our program, and compiled a ranking (Corona Immunitas 2020). While specificity was high for most tests, which is crucial when seroprevalence is low, there was evidence of limited sensitivity. Most validation studies are likely to be substantially biased (e.g., spectrum bias and differential verification bias) because of their designs according to recent systematic reviews (Corona Immunitas 2020; Deeks et al. 2020). Biased estimates of accuracy make the adjustment of seroprevalence estimates for (imperfect) sensitivity and specificity uncertain. Therefore, the members decided to use the SenASTrIS (Sensitive Anti-SARSCoV-2 Spike Trimer Immunoglobulin Serological) assay developed by the CHUV, the Swiss Federal Institute of Technology in Lausanne (EPFL) and the Swiss Vaccine Center as the common test (Fenwick et al. 2020). Reasons for this choice were the trimeric (entire S) target, the strong signal detected in persons with and without symptoms at the time of infection, the availability of $\operatorname{IgG}$ and $\operatorname{IgA}$, the specificity of $99.7 \%$ and no cross-reactive antibodies in sera from people infected with pre-pandemic coronaviruses, a high sensitivity of $96.6 \%$ post 15 days of infection, superior performance over commercial tests in population-based samples that missed up to $45 \%$ of seropositive persons, the possibility for quantification, the availability of a neutralizing antibody test, the evidence of superior performance in population-based samples in comparison with six commercial tests, the availability of test material, and the possibility of use of this Luminexbased test in other laboratories in Switzerland (Fenwick et al. 2020).

\section{Longitudinal follow-up}

A digital follow-up will be conducted for a duration of 6-12 months, depending on the progression of the pandemic, allowing for tracking the health status of individuals and for identifying new infections through reports of flulike symptoms. The digital follow-up consists of weekly, brief status updates with respect to self-reported symptoms 
and risk exposures as well as monthly follow-ups. Details on the weekly and monthly follow-ups are found in Table 3, and the questionnaires are in the supplementary material. In some centers, groups of participants will further be invited to participate in repeated in-person visits for testing of their SARS-CoV-2 antibody levels.

\section{Statistical analysis plan and sample size calculation}

There will be a centralized data management plan and a centralized process for analyses done on pooled data from all study sites. The primary endpoint for the overall study is the seroprevalence of SARS-CoV-2 antibodies in the general population at repeated time points during the pandemic in Switzerland. From the data collected within the seroprevalence studies of the randomly selected population sample, we will determine the age-specific and timespecific attack rate based on seroprevalence. We will also calculate the age-specific and region-specific cumulative incidence of seropositive individuals who have been asymptomatic, age-specific distribution of disease severity in seropositives who have been symptomatic, age-specific and disease severity-specific geometric mean level of IgG/ IgA antibody titers, and lastly population groups most at risk of being seropositive (e.g., age groups, sex, occupation). To estimate seroprevalence, we use a Bayesian logistic regression model, accounting for the age and sex of the general population, and weighted for the sampling strategy (Stringhini et al. 2020). We integrate this regression model with a binomial model of the antibody test sensitivity and specificity to adjust the estimates for test performance while propagating uncertainty around test performance into final seroprevalence estimates. Recent observations suggest that antibodies could decrease if not disappear with time (Ibarrondo et al. 2020; Long et al. 2020). If confirmed in larger and prospectively planned studies (see Table 2), we will account for this trend by modeling the impact on the estimated proportion of participants having been infected.

The association of seroprevalence with participants' characteristics, potential risk factors, and preventative measures will be assessed by mixed multiple linear and logistic regression analyses, with random effects for cantons, adjusted for potential confounders and will include interaction terms if reasonable. Analyses will be conducted in $\mathrm{R}$ ( $\mathrm{R}$ Core Team 2020), with some sites conducting local analyses using R or other software (e.g., STATA).

The sample size provides enough statistical power to estimate the seroprevalence of SARS-CoV-2 antibodies with reasonable accuracy in each Canton, for different age groups, and at different points in time (sample sizes range from $n=1200-8640$ per Canton; Table 1). Estimates are considered precise enough for informing policy-makers if the entire $90 \%$ credible interval leads to the same interpretation of seroprevalence and possible decisions. For specific groups of interest (e.g., age group $65+$ or any subpopulation), and considering a serological test with 98\% sensitivity and $99 \%$ specificity, a minimum population of 200 persons with an observed seroprevalence of $5 \%$ will yield $90 \%$ credible intervals of $\pm 2.5 \%$ for a posterior prevalence of $4.6 \%$, of $\pm 3.5 \%$ for a posterior seroprevalence of $9.7 \%$ (observed prevalence $10 \%$ ) and of $\pm 4.6 \%$ for a posterior seroprevalence of $19.9 \%$ (observed prevalence 20\%). We consider these precisions enough for informing policy-makers. Site are free to have larger samples per group of interest, and we will adapt the sample size calculations once the seroprevalence is higher than $20 \%$. For each age group, three sampling reserves (each representing $25 \%$ of the needed sample size) will additionally be drawn by the FSO. The reserves will be used in case of lower participation rate if the needed sample size is not attained.

\section{Results}

The University of Geneva provided phase one estimates for Geneva by enrolling 2766 participants from 1399 households with a demographic difference that mirrored the Canton. When they began in April 2020, the seroprevalence was estimated at $4.8 \%(95 \%$ CI 2.4-8.0, $n=341)$ (Stringhini et al. 2020). By the fifth week, the seroprevalence had risen to $10.8 \%$ (95\% CI 8.2-13.9, $n=775)$. The age group with highest risk of being seropositive was found to be those aged 20-49 years.

The centers of Zurich, Lausanne, Basel City/Land, Ticino, and Fribourg began recruitment for the second phase of the study and have recruited 5973 participants. Of those recruited, 1187, 1560, and 1677 participants have completed the first, second, and third weeks of the digital follow-up, respectively. A total of 900 participants have completed the first monthly follow-up.

Given the results from phase one from Geneva and the number of diagnosed cases of SARS-CoV-2 up to August 2020 across the Swiss Cantons, we expect declining seroprevalence rates from Southern to Northern and from Western to Eastern Switzerland. Thus, for phase two we may expect seroprevalences between 2 and 15\% across regions. For phases three (November 2020) and four (March 2021), this may change considerably because the pandemic now has substantially different numbers of new cases within German- and French-speaking cantons. 


\section{Discussion}

Corona Immunitas is a research program coordinated by SSPH+, conducting longitudinal, population-based seroprevalence studies covering a number of Swiss Cantons as well as several seroepidemiological studies in specific subpopulations. The population-based seroprevalence studies are conducted on population samples that are representative of the Cantonal populations, ensured by a random selection of residents in the Cantonal population registries. Studies on subpopulations cover various populations and are detailed in Table 2. Studies are mostly cross-sectional and a number of them include nested longitudinal components to help capture much more than serology estimates of the population.

Corona Immunitas addresses a number of limitations of the current evidence in seroprevalence studies. One goal is to conduct studies with low risk of bias and a sampling strategy for both general and subpopulations that reflect the target populations of interest as much as possible. We use an accurate serology test and consistent questionnaires across sites to give a clearer picture of the pandemic. The longitudinal component of the program will provide guidance as to the extent and duration of immunity, as well as the long-term impact of the pandemic and lockdown measures. Therefore, Corona Immunitas gives policymakers useful information for public health decisions. Several investigators of Corona Immunitas are members of the National COVID-19 Science Task Force of Switzerland that directly advises the FOPH and Federal Council (https://ncs-tf.ch/de/). Others are closely linked to cantonal health authorities and stakeholders. On the cantonal level, Corona Immunitas works with the respective health authorities and aims to establish a science to policy collaboration with the federation of Cantonal health directors. Finally, the program will provide information about the impact of the pandemic and the effectiveness of protective measures to decision makers for those persons with particularly exposed occupations and vulnerable persons.

Despite these efforts, there is potential for several limitations. First, even though efforts were made to recruit representative samples of the population by inviting randomly selected residents from cantonal population registries, we expect a relatively low participation rate which may introduce selection bias. Some reasons why individuals might not participate include lack of time and motivation, the fact that the assessment period of the second phase coincided with the Swiss summer holiday, the fear of being infected, and test fatigue (Bobrovitz et al. 2020). Other risks of selection bias that could artificially increase the estimated seroprevalence include stronger motivation to participate if they are symptomatic, were in contact with a person who tested positive for COVID-19, or travelled to a severely affected area. Although the coordination is strong on conceptual aspects of the study, practical aspects differ between study sites. Study sites share a common protocol, but some differences may still exist. To mitigate the risk that these differences impact on the results, the consortium will regularly compare SOPs and visit sites. Finally, antibodies may not be detectable in those who had a SARS-CoV-2 infection but with no or mild symptoms (Long et al. 2020) and estimates may therefore underestimate seroprevalence. However, the repeated serological testing will provide important insights into the potential disappearance of antibodies, factors associated with it and allow for correcting estimates of the proportion of people who had the infection.

One advantage of Corona Immunitas is to learn from other existing seroprevalence studies worldwide and the emergence of further evidence on the accuracy of serological tests as well as the nature of different tests. The use of a combined data management plan and nationwide serology test will guarantee interoperability and comparability. Additionally, the common work allows the consortium to cover different phases of the pandemic (https:// www.corona-immunitas.ch/program) and to study subpopulations in a complementary way, which avoids redundancies and increases exhaustiveness.

Another advantage of the Corona Immunitas program is the combined use of serological testing and questionnaires. Self-reported data on socioeconomic characteristics, symptoms, and contact tracing will give rise to future analyses, which will further the understanding of the pandemic in more depth than serological testing alone. It will provide the possibility to assess severity of illness per infection more accurately, as well as transmission dynamics, and effect of socioeconomic characteristics. Transmission parameters are important for assessing transmission risks and shaping sanitary recommendations tailored to workspaces, schools, or the private sphere, e.g., in households or for families, especially those with elderly parents in nursing homes (McMichael et al. 2020). Socioeconomic characteristics also need to be addressed, especially social determinants of health (Khalatbari-Soltani et al. 2020) and their relation with the disproportionate impact of death and severe illness on social minorities (Saini 2020). These populations can be disproportionately affected by negative consequences of the pandemic from a physical health, mental health and a socioeconomic perspective. Additionally, the subsequent digital follow-up will provide data to help nourish a deeper understanding of the pandemic and its related sanitary measures on mental and physical health, and overall well-being of the population and society. 
The Corona Immunitas program will inform on the prevalence of the population infected by region and its longitudinal components will inform on potential post-infection acquired immunity and its duration. Corona Immunitas is a unique nationwide research program that is centrally coordinated, which maintains the independence of all centers involved, ensuring both interoperability and comparability, and the adaptation of study designs to local needs. The Corona Immunitas consortium will generate reliable, comparable and high-quality data with extensive coverage of the Swiss geography and of several subpopulations of interest, to inform governmental and sectorspecific decision making on the management of the SARSCoV-2 pandemic. It can serve as a template for other regions and countries as it is important to have comparable data to fight this pandemic most efficiently.

Acknowledgements We thank the Swiss Federal Statistical Office for providing the randomized list of participants for the main populationbased portion of our study. We thank SSPH+ for their help with coordination of all study sites. We lastly wish to thank all nurses, medical students, researchers, clinicians, and staff that contributed to the implementation of Corona Immunitas.

Corona Immunitas Research Group The following are members of Corona Immunitas Research Group (including the authors of the present article), listed in alphabetical order: Emiliano Albanese, MD $\mathrm{PhD}$ (Institute of Public Health (IPH), Università della Svizzera Italiana (USI), Lugano, Switzerland; emiliano.albanese@usi.ch), Rebecca Amati, PhD (Institute of Public Health (IPH), Università della Svizzera Italiana (USI), Lugano, Switzerland; rebecca.amati@usi.ch), Antonio Amendola, Msc (Department of Business Economics, Health \& Social Care (DEASS), University of Applied Sciences \& Arts of Southern Switzerland (SUPSI), Manno, Switzerland; antonio.amendola@supsi.ch), Daniela Anker, Msc (Population Health Laboratory (\#PopHealthLab), University of Fribourg, Fribourg, Switzerland; Institute of Primary Health Care (BIHAM), University of Bern, Bern, Switzerland; daniela.anker@unifr.ch), Anna Maria Annoni, Msc (Institute of Public Health (IPH), Università della Svizzera Italiana (USI), Lugano, Switzerland; anna.maria.annoni@usi.ch), Andrew Azman, PhD (Division of Primary Care, Geneva University Hospitals, Geneva, Switzerland; Department of Epidemiology, Johns Hopkins Bloomberg School of Public Health, Baltimore, MD, USA; Institute of Global Health, University of Geneva, Geneva, Switzerland; andrew.azman@hcuge.ch), Frank Bally, MD (Institut central des hôpitaux, Hôpital du Valais, Sion, Switzerland ; frank.bally@hopitalvs.ch), Bettina Balmer, MD (Epidemiology, Biostatistics and Prevention Institute, University of Zurich, Zurich, Switzerland; balmer.schiltknecht@gmail.com), Hélène Baysson, PhD (Department of Health and Community Medicine, University of Geneva, Geneva, Switzerland; helene.baysson@unige.ch), Delphine Berthod, MD (Institut central des hôpitaux, Hôpital du Valais, Sion, Switzerland ; delphine.berthod@ hopitalvs.ch), Jacob Blankenberger, BSc (Epidemiology, Biostatistics and Prevention Institute, University of Zurich, Zurich, Switzerland; jacob.blankenberger@uzh.ch), Murielle Bochud, MD PhD (Center for Primary Care and Public Health (Unisanté), University of Lausanne, Lausanne, Switzerland, murielle.bochud@unisante.ch), Patrick Bodenmann, MD Msc (Center for Primary Care and Public Health (Unisanté), University of Lausanne, Lausanne, Switzerland; patrick.bodenmann@unisante.ch), Matthias Bopp, Dr. phil.II PhD MPH (Epidemiology, Biostatistics and Prevention Institute, University of Zurich, Zurich, Switzerland; matthias.bopp@uzh.ch), Audrey Butty,
MD (Center for Primary Care and Public Health (Unisanté), University of Lausanne, Lausanne, Switzerland; audrey.butty@unisante.ch), Anne Linda Camerini, PhD (Institute of Public Health (IPH), Università della Svizzera Italiana (USI), Lugano, Switzerland; anne.linda.camerini@usi.ch), Céline Cappeli, BSc (Epidemiology, Biostatistics and Prevention Institute, University of Zurich, Zurich, Switzerland; celine.capelli@uzh.ch), Cristian Carmelli, PhD (Population Health Laboratory (\#PopHealthLab), University of Fribourg, Fribourg, Switzerland; cristian.carmeli@unifr.ch), Arnaud Chiolero, MD PhD, (Population Health Laboratory (\#PopHealthLab), University of Fribourg, Fribourg, Switzerland; Institute of Primary Health Care (BIHAM), University of Bern, Bern, Switzerland; Department of Epidemiology, Biostatistics and Occupational Health, McGill University, Montréal, Canada; arnaud.chiolero@unifr.ch), Prune Collombet, MSc (Division of Primary Care, Geneva University Hospitals, Geneva, Switzerland; prune.collombet@hcuge.ch), Laurie Corna, PhD (Department of Business Economics, Health \& Social Care (DEASS), University of Applied Sciences \& Arts of Southern Switzerland (SUPSI), Manno, Switzerland; laurie.corna@supsi.ch), Jenny Crawford, MPH (Epidemiology, Biostatistics and Prevention Institute, University of Zurich, Zurich, Switzerland; jenny.crawford@uzh.ch), Luca Crivelli, PhD (Department of Business Economics, Health \& Social Care (DEASS), University of Applied Sciences \& Arts of Southern Switzerland (SUPSI), Manno, Switzerland; Institute of Public Health (IPH), Università della Svizzera Italiana (USI), Lugano, Switzerland; luca.crivelli@supsi.ch), Stéphane Cullati, PhD (Population Health Laboratory (\#PopHealthLab), University of Fribourg, Fribourg, Switzerland; Department of Readaptation and Geriatrics, Faculty of Medicine, University of Geneva, Switzerland; stephane.cullati@unifr.ch), Alexia Cusini, MD (Infectious Diseases Unit, Cantonal Hospital, 7000 Chur, Switzerland; alexia.cusini@ksgr.ch), Valérie D’Acremont, MD PhD (Center for Primary Care and Public Health (Unisanté), University of Lausanne, Lausanne, Switzerland; Swiss Tropical and Public Health Institute, University of Basel, Basel, Switzerland; valerie.dacremont@unisante.ch), Carlo De Pietro, PhD (Department of Business Economics, Health \& Social Care (DEASS), University of Applied Sciences \& Arts of Southern Switzerland (SUPSI), Manno, Switzerland; carlo.depietro@supsi.ch), Agathe Deschamps, MPH (Cantonal Medical Service, Neuchâtel, Switzerland; agathe.deschamps@ne.ch), Yaron Dibner (Population Epidemiology Unit, Primary Care Division, Geneva University Hospitals, Geneva, Switzerland; yaron.diber@hcuge.ch), Sophie Droz, BA (Cantonal Medical Service, Neuchâtel, Switzerland; sophie.droz@ne.ch), Alexis Dumoulin, PhD (Institut central des hôpitaux, Hôpital du Valais, Sion, Switzerland ; alexis.dumoulin@hopitalvs.ch), Olivier Duperrex, MD Msc (Center for Primary Care and Public Health (Unisanté), University of Lausanne, Lausanne, Switzerland; olivier.duperrex@unisante.ch), Julien Dupraz, MD MAS (Center for Primary Care and Public Health (Unisanté), University of Lausanne, Lausanne, Switzerland; julien.dupraz@unisante.ch), Malik Egger, MSc (Center for Primary Care and Public Health (Unisanté), University of Lausanne, Lausanne, Switzerland; malik.egger@unisante.ch), Nathalie Engler, (Cantonal Hospital St. Gallen, Clinic for Infectious Diseases and Hospital Epidemiology, St. Gallen, Switzerland; natalie.engler@kssg.ch), Adina Mihaela Epure, MD (Population Health Laboratory (\#PopHealthLab), University of Fribourg, Fribourg, Switzerland; Department of Epidemiology and Health Services, Center for Primary Care and Public Health (Unisanté), University of Lausanne, Lausanne, Switzerland; adina-mihaela.epure@unifr.ch), Sandrine Estoppey, MSc (Center for Primary Care and Public Health (Unisanté), University of Lausanne, Lausanne, Switzerland; sandrine.estoppey@unisante.ch), Marta Fadda, PhD (Institute of Public Health (IPH), Università della Svizzera Italiana (USI), Lugano, Switzerland; marta.fadda@usi.ch), Vincent Faivre (Center for Primary Care and Public Health (Unisanté), University of Lausanne, 
Lausanne, Switzerland; vincent.faivre@unisante.ch), Jan Fehr, MD (Epidemiology, Biostatistics and Prevention Institute, University of Zurich, Zurich, Switzerland; jan.fehr@usz.ch), Andrea Felappi (Center for Primary Care and Public Health (Unisanté), University of Lausanne, Lausanne, Switzerland; andrea.felappi@unisante.ch), Maddalena Fiordelli, PhD (Institute of Public Health (IPH), Università della Svizzera Italiana (USI), Lugano, Switzerland; maddalena.fiordelli@usi.ch), Antoine Flahault,.MD PhD (Institute of Global Health, Faculty of Medicine, University of Geneva, Geneva, Switzerland; Division of Tropical and Humanitarian Medicine, Geneva University Hospitals, Geneva, Switzerland; Department of Health and Community Medicine, University of Geneva, Geneva, Switzerland; antoine.flahault@unige.ch), Luc Fornerod, MAS (Observatoire valaisan de la santé (OVS), Sion, Switzerland ; luc.fornerod@ovs.ch), Cristina Fragoso Corti, $\mathrm{PhD}$ (Department of environment construction and design (DACD), University of Applied Sciences \& Arts of Southern Switzerland (SUPSI), Manno, Switzerland; cristina.fragoso@supsi.ch), Marion Frangville, Msc (Division of Primary Care, Geneva University Hospitals, Geneva, Switzerland; marion.frangville@hcuge.ch), Irène Frank, PhD (Clinical Trial Unit, Cantonal Hospital Luzern, Luzern, Switzerland; irene.frank@luks.ch) Giovanni Franscella, Msc (Institute of Public Health (IPH), Università della Svizzera Italiana (USI), Lugano, Switzerland; giovanni.franscella@usi.ch), Anja Frei, PhD (Epidemiology, Biostatistics and Prevention Institute, University of Zurich, Zurich, Switzerland; anja.frei@uzh.ch), Doreen Gille, MSc (Epidemiology, Biostatistics and Prevention Institute, University of Zurich, Zurich, Switzerland; doreen.gille@uzh.ch), Gisela Michel, PhD (Department of Health Sciences and Medicine, University of Luzern, Luzern, Switzerland; gisela.michel@unilu.ch)Semira Gonseth Nusslé, MSc MD (Center for Primary Care and Public Health (Unisanté), University of Lausanne, Lausanne, Switzerland; semira.gonseth-nussle@unisante.ch), Auriane Gouzowski, MA (Cantonal Medical Service, Neuchâtel, Switzerland; Auriane.gouzowski@ne.ch), Idris Guessous, MD, PhD (Division of Primary Care, Geneva University Hospitals, Geneva, Switzerland; Department of Health and Community Medicine, Faculty of Medicine, University of Geneva, Geneva, Switzerland; idris.guessous@hcuge.ch), Julien Guggisberg, BA (Cantonal Medical Service, Neuchâtel, Switzerland; Julien.guggisberg@ne.ch), Huldrych Günthard, MD (Epidemiology, Biostatistics and Prevention Institute, University of Zurich, Zurich, Switzerland; huldrych.guenthard@usz.ch), Felix Gutzwiller, MD, PhD (Epidemiology, Biostatistics and Prevention Institute, University of Zurich, Zurich, Switzerland), Medea Imboden, PhD (Swiss Tropical and Public Health Institute, Basel, Switzerland, University of Basel, Basel, Switzerland; medea.imboden@swisstph.ch), Loussine Incici, LLM(Cantonal Medical Service, Neuchâtel, Switzerland; loussine.incici@ne.ch), Emilie Jendly (Center for Primary Care and Public Health (Unisanté), University of Lausanne, Lausanne, Switzerland; emilie.jendly@unisante.ch), Ruedi Jung, Msc (Epidemiology, Biostatistics and Prevention Institute, University of Zurich, Zurich, Switzerland; ruedi.jung@uzh.ch), Christian Kahlert, MD (Cantonal Hospital St. Gallen, Clinic for Infectious Diseases and Hospital Epidemiology, St. Gallen, Switzerland; Children's Hospital of Eastern Switzerland, Infectious Diseases and Hospital Epidemiology, St. Gallen, Switzerland; christian.kahlert@kssg.ch), Laurent Kaiser, MD PhD (Geneva Center for Emerging Viral Diseases and Laboratory of Virology, Geneva University Hospitals, Geneva, Switzerland; Division of Infectious Diseases, Geneva University Hospitals, Geneva, Switzerland; Department of Medicine, Faculty of Medicine, University of Geneva, Geneva, Switzerland; laurent.kaiser@hcuge.ch), Laurent Kaufmann, MD (Cantonal Medical Service, Neuchâtel, Switzerland; laurent.kaufmann@ne.ch), Marco Kaufmann, $\mathrm{PhD}$ (Epidemiology, Biostatistics and Prevention Institute, University of Zurich, Zurich, Switzerland; marco.kaufmann@uzh.ch), Simone Kessler, (Cantonal Hospital St. Gallen,
Clinic for Infectious Diseases and Hospital Epidemiology, St. Gallen, Switzerland; simone.kessler@kssg.ch), Philipp Kohler, MD MSc (Cantonal Hospital St. Gallen, Clinic for Infectious Diseases and Hospital Epidemiology, St. Gallen, Switzerland; philipp.kohler@kssg.ch), Susi Kriemler, MD (Epidemiology, Biostatistics and Prevention Institute, University of Zurich, Zurich, Switzerland; susi.kriemlerwiget@uzh.ch), Lauranne Lenoir, MA (Cantonal Medical Service, Neuchâtel, Switzerland; lauranne.lenoir@ne.ch), Sara Levati, PhD (Department of Business Economics, Health \& Social Care (DEASS), University of Applied Sciences \& Arts of Southern Switzerland (SUPSI), Manno, Switzerland; sara.levati@supsi.ch), Bettina Maeschli (Epidemiology, Biostatistics and Prevention Institute, University of Zurich, Zurich, Switzerland; maeschli@corona-immunitas.ch), Jean-Luc Magnin, PhD (Laboratory, HFR-Fribourg, Fribourg, Switzerland; jean-luc.magnin@h-fr.ch), Eric Masserey, MD (Cantonal Medical Office, General Health Department, Canton of Vaud, Lausanne, Switzerland; eric.masserey@vd.ch), Rosalba Morese, PhD (Institute of Public Health (IPH), Università della Svizzera Italiana (USI), Lugano, Switzerland; rosalba.morese@usi.ch), Nicolai Mösli, MD (Swiss Tropical and Public Health Institute, University of Basel, Basel, Switzerland; nicolai.moesli@swisstph.ch), Natacha Noël (Population Epidemiology Unit, Primary Care Division, Geneva University Hospitals, Geneva, Switzerland; natacha.noel@hcuge.ch), Maëlle Orhant, BA (Cantonal Medical Service, Neuchâtel, Switzerland), Daniel Henry Paris, MD PhD (Swiss Tropical and Public Health Institute, University of Basel, Basel, Switzerland; daniel.paris@swisstph.ch), Jérôme Pasquier, PhD (Center for Primary Care and Public Health (Unisanté), University of Lausanne, Lausanne, Switzerland; jerome.pasquier@unisante.ch), Francesco Pennacchio, $\mathrm{PhD}$ (Division of Primary Care, Geneva University Hospitals, Geneva, Switzerland; francesco.pennacchio@hcuge.ch), Dusan Petrovic, $\mathrm{PhD}$ (Division of Primary Care, Geneva University Hospitals, Geneva, Switzerland; dusan.petrovic@hcuge.ch), Stefan Pfister, PhD (Laboratory, HFR-Fribourg, Fribourg, Switzerland; stefan.pfister@hfr.ch), Attilio Picazio, PhD (Population Epidemiology Unit, Primary Care Division, Geneva University Hospitals, Geneva, Switzerland), Cesarina Prandi, PhD (Department of Business Economics, Health \& Social Care (DEASS), University of Applied Sciences \& Arts of Southern Switzerland (SUPSI), Manno, Switzerland; cesarina.prandi@supsi.ch); Giovanni Piumatti, PhD (Institute of Public Health (IPH), Università della Svizzera Italiana (USI), Lugano, Switzerland; Giovanni.Piumatti@unige.ch), Jane Portier (Department of Primary Care, Geneva University Hospitals, Geneva, Switzerland ; jane.portier@hcuge.ch), Nicole Probst-Hensch, Dr. phil.II PhD MPH (Swiss Tropical and Public Health Institute, University of Basel, Basel, Switzerland; nicole.probst@swisstph.ch), Caroline Pugin (Population Epidemiology Unit, Primary Care Division, Geneva University Hospitals, Geneva, Switzerland; caroline.pugin@hcuge.ch), Milo Puhan, MD PhD (Epidemiology, Biostatistics and Prevention Institute, University of Zurich, Zurich, Switzerland; miloalan.puhan@uzh.ch), Thomas Radtke, PhD (Epidemiology, Biostatistics and Prevention Institute, University of Zurich, Zurich, Switzerland; thomas.radtke@uzh.ch), Aude Richard, MD MPH (Division of Primary Care, Geneva University Hospitals, Geneva, Switzerland; Institute of Global Health, Faculty of Medicine, University of Geneva, Geneva, Switzerland; aude.richard@unige.ch), Claude-François Robert, MD (Cantonal Medical Service, Neuchâtel, Switzerland; claude-francois.robert@ne.ch), Pierre-Yves Rodondi, MD (Institute of Family Medicine, University of Fribourg, Fribourg, Switzerland; pierre-yves.rodondi@unifr.ch), Nicholas Rodondi, MD (Institute of Primary Health Care (BIHAM), University of Bern, Switzerland; nicholas.rodondi@biham.unibe.ch), Eric Salberg, BA (Cantonal Medical Service, Neuchâtel, Switzerland; Eric.salberg@ne.ch), Javier Sanchis Zozaya, MD (Center for Primary Care and Public Health (Unisanté), University of Lausanne, Lausanne, 
Switzerland; javier.sanchis-zozaya@unisante.ch), Virginie Schlüter, MAS, MD (Center for Primary Care and Public Health (Unisanté), University of Lausanne, Lausanne, Switzerland; virginie.schlueter@unisante.ch), Valentine Schneider, MSc (Cantonal Medical Service, Neuchâtel, Switzerland; valentine.schneider@ne.ch), Amélie Steiner-Dubuis (Center for Primary Care and Public Health (Unisanté), University of Lausanne, Switzerland; amelie.steiner-dubuis@unisante.ch), Silvia Stringhini, PhD (Division of Primary Care, Geneva University Hospitals, Geneva, Switzerland; silvia.stringhini@hcuge.ch), Johannes Sumer, MD (Cantonal Hospital St. Gallen, Clinic for Infectious Diseases and Hospital Epidemiology, St. Gallen, Switzerland; johannes.sumer@kssg.ch), Ismaël Tall, MA (Cantonal Medical Service, Neuchâtel, Switzerland; Ismael.tall@ne.ch), Julien Thabard (Center for Primary Care and Public Health (Unisanté), University of Lausanne, Lausanne, Switzerland; julien.thabard@unisante.ch), Mauro Tonolla, PhD (Department of environment construction and design (DACD), University of Applied Sciences \& Arts of Southern Switzerland (SUPSI), Manno, Switzerland; mauro.tonolla@supsi.ch), Nicolas Troillet, MD Msc (Institut central des hôpitaux, Hôpital du Valais, Sion, Switzerland ; nicolas.troillet@hopitalvs.ch), Agne Ulyte, MD (Epidemiology, Biostatistics and Prevention Institute, University of Zurich, Zurich, Switzerland; agne.ulyte@uzh.ch), Sophie Vassaux, MSc (Center for Primary Care and Public Health (Unisanté), University of Lausanne, Lausanne, Switzerland; sophie.vassaux@unisante.ch), Thomas Vermes, Msc (Swiss Tropical and Public Health Institute, University of Basel, Basel, Switzerland; thomas.vermes@swisstph.ch), Fabian Vollrath, Msc, (Epidemiology, Biostatistics and Prevention Institute, University of Zurich, Zurich, Switzerland; vollrath@corona-immunitas.ch),Viktor von Wyl, PhD (Epidemiology, Biostatistics and Prevention Institute, University of Zurich, Zurich, Switzerland; viktor.vonwyl@uzh.ch), Erin West, Msc (Epidemiology, Biostatistics and Prevention Institute, University of Zurich, Zurich, Switzerland; erinashley.west@uzh.ch), Ania Wisniak, MD (Division of Primary Care, Geneva University Hospitals, Geneva, Switzerland; Institute of Global Health, Faculty of Medicine, University of Geneva, Geneva, Switzerland; anna.wisniak@unige.ch), Maria-Eugenia Zaballa, PhD (Population Epidemiology Unit, Primary Care Division, Geneva University Hospitals, Geneva, Switzerland; mariaeugenia.zaballa@hcuge.ch), Claire Zuppinger, MSc (Center for Primary Care and Public Health (Unisanté), University of Lausanne, Lausanne, Switzerland; claire.zuppinger@unisante.ch)

Author contributions MAP, EA, MB, AC, LC, SC, Vd'A, LK, AME, JF, AF, LF, GM, SG, IG, MI, CRK, PK, NM, DP, NPH, NR, SS, and TV contributed to the conception and design of the study. AF provided support to sites for ethics approvals. FV provided managerial and coordination assistance to all sites. RA, AR, AW, and $A B$ provided information on cantonal substudies. EAW and DA drafted the manuscript and all commented on it.

Funding Open access funding provided by University of Zurich. The directorate of SSPH+ is responsible for the coordination, communication, fundraising, and legal aspects of the population-based studies and the central program of Corona Immunitas. SSPH+ conducts extensive fundraising from public (e.g., FOPH) and private sources (foundations, companies, and private donations) with the option for sites to have additional sources for their subpopulation studies. Donors have no influence on the design, conduct, analyses and publications. A summary of these details per site is in the supplementary material Table S1. This study was funded by several sources (full funding details in Supplementary Materials) that includes, but is not limited to, SSPH+ and Swiss Federal Office of Public Health.

\section{Compliance with ethical standards}

Conflict of interest The authors declare that they have no conflict of interest.

Ethical approval The Ethics Committees of the various cantons approve this study (BASEC 2020-01247).

Informed consent The subjects of the study were provided informed consent (included in submission) prior to their participation in the study.

Open Access This article is licensed under a Creative Commons Attribution 4.0 International License, which permits use, sharing, adaptation, distribution and reproduction in any medium or format, as long as you give appropriate credit to the original author(s) and the source, provide a link to the Creative Commons licence, and indicate if changes were made. The images or other third party material in this article are included in the article's Creative Commons licence, unless indicated otherwise in a credit line to the material. If material is not included in the article's Creative Commons licence and your intended use is not permitted by statutory regulation or exceeds the permitted use, you will need to obtain permission directly from the copyright holder. To view a copy of this licence, visit http://creativecommons. org/licenses/by/4.0/.

\section{References}

Arora RK, Joseph A, Van Wyk J, Rocco S, Atmaja A, May E et al (2020) (2020) SeroTracker: a global SARS-CoV-2 seroprevalence dashboard. Lancet Infect Dis 3099(20):9-10. https://doi. org/10.1016/S1473-3099(20)30631-9

Bobrovitz N, Arora RK, Yan T, Rahim H, Duarte N, Boucher E et al (2020) Lessons from a rapid systematic review of early SARSCoV-2 serosurveys. medRxiv. https://doi.org/10.1101/2020.05. 10.20097451

Corona Immunitas (2020) Choosing a common test. https://www.de. corona-immunitas.ch/blog/choosing-a-common-test. Accessed 21 July 2020

de Mestral C, Stringhini S, Guessous I, Jornayvaz FR (2019) Thirteen-year trends in the prevalence of diabetes in an urban region of Switzerland: a population-based study. Diabet Med 2019:1374-1378

Deeks JJ, Dinnes J, Takwoingi Y, Davenport C, Spijker R, TaylorPhillips S et al (2020) (2020) Antibody tests for identification of current and past infection with SARS-CoV-2. Cochrane Database Syst Rev 6:CD013652

Fenwick F, Croxatto A, Coste AT, Pojer F, André C, Pellaton C et al (2020) Changes in SARS-CoV-2 antibody responses impact the estimates of infections in population-based seroprevalence studies. medRxiv. https://doi.org/10.1101/2020.07.14. 20153536v2

Gorbalenya AE, Baker SC, Baric RS, de Groot RJ, Drosten C, Gulyaeva AA et al (2020) The species Severe acute respiratory syndrome-related coronavirus: classifying 2019-nCoV and naming it SARS-CoV-2. Nat Microbiol Nat Res. https://doi.org/10. 1038/s41564-020-0695z

Harris PA, Taylor R, Minor BL, Elliott V, Fernandez M, O’Neal L et al (2019) The REDCap consortium: building an international community of software platform partners. J Biomed Inform 95:103208 
Hellewell J, Abbott S, Gimma A, Bosse NI, Jarvis CI, Russell TW et al (2020) Feasibility of controlling COVID-19 outbreaks by isolation of cases and contacts. Lancet Glob Heal 8(4):e488 e496. https://doi.org/10.1016/S2214-109X(20)30074-7

Horby PW, Laurie KL, Cowling BJ, Engelhardt OG, Sturm-Ramirez K, Sanchez JL et al (2017) CONSISE statement on the reporting of seroepidemiologic studies for influenza (ROSES-I statement): an extension of the STROBE statement. Influenza Other Respi Viruses 11(1):2-14

Ibarrondo FJ, Fulcher JA, Goodman-Meza D, Elliott J, Hofmann C, Hausner MA et al (2020) Rapid decay of Anti-SARS-CoV-2 antibodies in persons with mild Covid-19. N Engl J Med. https:// doi.org/10.1056/NEJMc2025179

Joanna Briggs Institute (2016) Critical appraisal checklist for systematic reviews and research syntheses. Joanna Briggs Institute critical appraisal tools for use in JBI systematic reviews. 13(3): 1-7

Khalatbari-Soltani S, Cumming RC, Delpierre C, Kelly-Irving M (2020) Importance of collecting data on socioeconomic determinants from the early stage of the COVID-19 outbreak onwards. J Epidemiol Community Health. https://doi.org/10. 1136/jech-20200214297

Long QX, Tang XJ, Shi QL, Li Q, Deng HJ, Yuan J et al (2020) Clinical and immunological assessment of asymptomatic SARSCoV-2 infections. Nat Med. https://doi.org/10.1038/s41591-0200965-6

McMichael TM, Currie DW, Clark S, Pogosjans S, Kay M, Schwartz NG et al (2020) Epidemiology of covid-19 in a long-term care facility in King County, Washington. $N$ Engl J Med 382(21):2008-2011

Pollán M, Pérez-Gómez B, Pastor-Barriuso R, Oteo J, Hernán MA, Pérez-Olmeda M et al (2020) Prevalence of SARS-CoV-2 in Spain (ENE-COVID): a nationwide, population-based seroepidemiological study. Lancet (London, England). https://doi.org/ 10.1016/S0140-6736(20)31483-5

R Core Team (2020) R: A language and environment for statistical computing. R Foundation for Statistical Computing, Vienna, Austria. ISBN 3-900051-07-0. https://www.R-project.org/. Accessed 21 July 2020

Saini A (2020) Stereotype threat. Lancet 395(10237):1604-1605

Stringhini S (2020) Unité d'épidémiologie populationnelle (UEP) Hôpitaux Universitaires Genève. https://www.hug.ch/medecinepremier-recours/unite-epidemiologie-populationnelle. Accessed 21 July 2020
Stringhini S, Wisniak A, Piumatti G, Azman AS, Lauer SA, Baysson $\mathrm{H}$ et al (2020) (2020) Seroprevalence of anti-SARS-CoV-2 IgG antibodies in Geneva, Switzerland (SEROCoV-POP): a population-based study. Lancet 6736(20):1-7

Swiss Federal Office of Public Health (2020a) Coronavirus: Federal Council declares 'extraordinary situation' and introduces more stringent measures. https://www.bag.admin.ch/bag/en/home/dasbag/aktuell/medienmitteilungen.msg-id-78454.html. Accessed 7 July 2020

Swiss Federal Office of Public Health (2020b) New coronavirus: people at especially high risk. https://www.bag.admin.ch/bag/en/ home/krankheiten/ausbrueche-epidemien-pandemien/aktuelleausbrueche-epidemien/novel-cov/besonders-gefaehrdetemenschen.html. Accessed 28 June 2020

Swiss Federal Office of Public Health (2020c) New coronavirus: situation in Switzerland. https://www.bag.admin.ch/bag/en/ home/krankheiten/ausbrueche-epidemien-pandemien/aktuelleausbrueche-epidemien/novel-cov/situation-schweiz-und-interna tional.html. Accessed 21 Aug 2020

Swiss Federal Office of Public Health (2020d) New coronavirus: SwissCovid app and contact tracing. https://www.bag.admin.ch/ bag/en/home/krankheiten/ausbrueche-epidemien-pandemien/ aktuelle-ausbrueche-epidemien/novel-cov/swisscovid-app-undcontact-tracing.html. Accessed 20 July 2020

Swiss Society of Hypertension (2020) Stellungnahme der Schweizerischen Hypertonie Gesellschaft (SHG) zur arteriellen Hypertonie und der COVID-19 Infektion (19.3.2020). https://www. swisshypertension.ch/DOCS_PUBLIC/Stellungnahme_COVID_ 19 D.pdf. Accessed 20 July 2020

Webmeter (2020) Coronavirus age, sex, demographics (COVID19)—Worldometer. www.worldometers.info. Accessed 21 Aug 2020

World Health Organization (2013) Guidance on conducting serosurveys in support of measles and rubella elimination in the WHO European Region. https://www.euro.who.int/_data/assets/pdf_ file/0011/236648/Guidance-on-conducting-serosurveys-in-sup port-of-measles-and-rubella-elimination-in-the-WHO-EuropeanRegion.pdf?ua=1. Accessed 20 July 2020

World Health Organization (2020) Population-based age-stratified seroepidemiological investigation protocol for COVID-19 virus infection. World Heal Organ. 2020;(March):1-19

Publisher's Note Springer Nature remains neutral with regard to jurisdictional claims in published maps and institutional affiliations.

\section{Affiliations}

Erin A. West ${ }^{1} \cdot$ Daniela Anker $^{2} \cdot$ Rebecca Amati $^{3} \cdot$ Aude Richard $^{4,5} \cdot$ Ania Wisniak $^{4,5} \cdot$ Audrey Butty $^{6}$. Emiliano Albanese $^{3}$ - Murielle Bochud ${ }^{6}$ - Arnaud Chiolero ${ }^{2,7,8}$ - Luca Crivelli ${ }^{3,9}$ - Stéphane Cullati ${ }^{2,10}$. Valérie d'Acremont ${ }^{6,11,12}$. Adina Mihaela Epure ${ }^{2,6} \cdot \mathrm{Jan} \mathrm{Fehr}^{1} \cdot$ Antoine Flahault $^{5} \cdot$ Luc Fornerod $^{13}$. Irène Frank ${ }^{14}$ - Anja Frei ${ }^{1}$ - Gisela Michel ${ }^{15}$. Semira Gonseth ${ }^{6}$. Idris Guessous ${ }^{4}$ - Medea Imboden ${ }^{11,12}$. Christian R. Kahlert $^{16,17}$ - Laurent Kaufmann ${ }^{18}$ - Philipp Kohler ${ }^{16}$ - Nicolai Mösli ${ }^{11,12}$ - Daniel Paris ${ }^{11,12}$. Nicole Probst-Hensch ${ }^{11,12} \cdot$ Nicolas Rodondi $^{7,19} \cdot$ Silvia Stringhini $^{4,6} \cdot$ Thomas Vermes $^{11,12} \cdot$ Fabian Vollrath $^{20}$. Milo A. Puhan ${ }^{1}$ the Corona Immunitas Research Group the Corona Immunitas Research Group

1 Epidemiology, Biostatistics and Prevention Institute, University of Zurich, Hirschengraben 84, 8001 Zurich, Switzerland

2 Population Health Laboratory (\#PopHealthLab), University of Fribourg, Fribourg, Switzerland
Institute of Public Health, Università Della Svizzera Italiana, Lugano, Switzerland

4 Unit of Population Epidemiology, Division of Primary Care Medicine, Geneva University Hospitals, Geneva, Switzerland 
5 Institute of Global Health, Faculty of Medicine, University of Geneva, Geneva, Switzerland

6 Center for Primary Care and Public Health (Unisanté), University of Lausanne, Lausanne, Switzerland

7 Institute of Primary Health Care (BIHAM), University of Bern, Bern, Switzerland

8 Department of Epidemiology, Biostatistics, and Occupational Health, McGill University, Montreal, Canada

9 Department of Business Economics, Health and Social Care, University of Applied Sciences and Arts of Southern Switzerland, Manno, Switzerland

10 Department of Readaptation and Geriatrics, Faculty of Medicine, University of Geneva, Geneva, Switzerland

11 Swiss Tropical and Public Health Institute, Basel, Switzerland

12 University of Basel, Basel, Switzerland
13 Health Observatory of Valais, Sion, Switzerland

14 Clinical Trial Unit, Cantonal Hospital Luzern, Luzern, Switzerland

15 Department of Health Sciences and Medicine, University of Luzern, Luzern, Switzerland

16 Department of Infectious Diseases and Hospital Epidemiology, Cantonal Hospital St. Gallen, St. Gallen, Switzerland

17 Infectious Diseases and Hospital Epidemiology, Children's Hospital of Eastern Switzerland, St. Gallen, Switzerland

18 Service de La Santé Publique, Canton de Neuchâtel, Neuchâtel, Switzerland

19 Department of General Internal Medicine, Inselspital, Bern University Hospital, University of Bern, Bern, Switzerland

20 Corona Immunitas Program Management Group, Swiss School of Public Health, Zurich, Switzerland 